\title{
Nutzung von Absatzkanälen für Wein - eine analyse aus Sicht deutscher Konsumenten
}

\section{Usage of sales channels for wine - an analysis from the perspective of German wine consumers}

\author{
G. Szolnoki \\ Geisenheim University, Von-Lade-Str. 1, 65366 Geisenheim, Germany
}

\begin{abstract}
Zusammenfassung. Die Bedeutung von Wein für Einkaufsstätten ist seit langem bekannt. Dementsprechend wird Wein als Zugkraft angewendet, um Kunden anzuziehen und Konsumenten mit tollen Angeboten sowie einem breiten Sortiment an sich zu binden. In Deutschland, wo jährlich mehr als 20 Mio. hl Wein vermarktet werden, ist die Struktur und Wertschöpfung von Einkaufsstätten von großer Bedeutung. Für die Analyse der wirtschaftlichen Bedeutung unterschiedlicher Einkaufsstätten wurden Daten einer Repräsentativbefragung von 2.000 Konsumenten herangezogen. Mithilfe eines entwickelten Modells konnten Eigenschaften wie Marktdurchdringung, Mengen- und Wertanteil errechnet werden. Einkaufstätten ohne Bedienungsservice machen mengenmäßig ca. 2/3 des Gesamtmarktes aus. Dies bedeutet, dass zwei von drei Flaschen im Handel über Discounter oder Supermärkte bzw. den Großhandel abgesetzt werden. Der Anteil von Fachgeschäften und Ab-Hof-Verkauf beläuft sich insgesamt auf $1 / 3$ der vermarkteten Gesamtmenge. Aufgrund der hier ermittelten Mengen und Preise lässt sich ein Durchschnittspreis für den Gesamtweinmarkt in Deutschland von $4.99 € / 1$ errechnen. Basierend auf den oben genannten Mengen und Werten wird der Gesamtumsatz von Stillwein im deutschen Handel auf 7.0 Mrd. €geschätzt. Da zu vermuten ist, dass Untergruppen wie Männer/Frauen und jüngere/ältere Konsumenten ihre Weine auf unterschiedliche Art und Weise beziehen, wurde auch segmentspezifisch analysiert, welche Untergruppen welche Einkaufsstätten für ihren Weinkauf präferieren.
\end{abstract}

\begin{abstract}
Sales channels have recognised long time ago the attraction of wine - wine brings new consumers - and they try to build customer loyalty with great offers and a wide range of products. In Germany, where more than 20 million hl of wine are marketed annually, the structure, volume and value of wine in different types of sales channels is of great importance. In order to quantify the economic importance of these sales channels, data from a representative survey of 2,000 consumers were used. The developed model helped calculate characteristics such as market penetration, quantity and value. Discount stores, supermarkets and wholesalers account for about $2 / 3$ of the total market. The share of specialized wine stores and direct sales at the winery (cellar door) totalled $1 / 3$ of the quantity marketed. Based on the volume and value determined in the model, an average price for the total wine market in Germany of $4.99 € / 1$ can be calculated and the total turnover of still wine in the German off-trade sector is estimated at $€ 7.0$ billion. Since it can be assumed that subgroups such as man/women and younger/older consumers purchase their wines in different ways, a segment-specific analysis was also carried out to find out which subgroups prefer which sales channels for their wine purchases.
\end{abstract}

\section{Einführung}

Für Konsumenten ist Wein ein Genussprodukt mit emotionalen Dimensionen. Einkaufsstätten haben vor langer Zeit den Magnet-Effekt dieses Produktes erkanntWein bringt neue Kunden - und so versuchen sie die Konsumenten mit tollen Angeboten und einem breiten Sortiment an sich zu binden. Die Strategie der größeren Einkaufsstätten-Ketten ist logisch nachvollziehbar: Mit Wein können sie Bestandskunden noch stärker an sich binden und haben gleichzeitig die Möglichkeit, ganz neue Verbrauchersegmente anzusprechen, die vorher gar nicht zum Klientel gehörten. In den letzten Jahren löste die Listung neuer Weine und somit die Erweiterung und
Neustrukturierung des Sortimentes sowie das Bestreben einen erlebnisorientierten Weinkauf anzubieten, eine leise, jedoch spürbare Revolution bei den verschiedenen Einkaufstätten-Typen aus. Discounter nahmen Weine in ihr Portfolio auf, die früher unvorstellbar waren und was einer Kampfansage an die Supermärkte gleichkam. Parallel dazu erweiterten viele größere, klassische Supermärkte ihre Beratung und sonstige Serviceangebote im Bereich Wein. Damit haben sie für viele Konsumenten die Rolle der spezialisierten Fachgeschäfte übernommen und dadurch mehr Kunden gewonnen.

Im Kampf um die Konsumenten hat sich in den letzten Jahren die Bedeutung der Einkaufsstätten bezüglich Wein geändert. Dieser Artikel beschreibt zunächst, 
wie häufig die verschiedenen alkoholischen Getränke konsumiert werden und analysiert die Nutzung der Einkaufsstätten sowie die Unterschiede zwischen den Weinkäufer-Segmenten. Darüber hinaus wird hier ein Modell dargestellt, das den deutschen Gesamtweinmarkt mengen- und wertmäßig beschreibt (Szolnoki, 2019).

\section{Material und methode}

Im Rahmen einer Mehrthemenumfrage zu Wein wurden im April 2018 auf der Basis einer zufällig ausgewählten Stichprobe von 2.063 Personen, die repräsentativ für die soziodemografische Struktur der Bevölkerung ab 16 Jahren in Deutschland ist, mittels eines vollstrukturierten Fragebogens verschiedene Fragen zur Nutzung, zum Einkauf und zur Präferenz für Wein erhoben.

Die Auswahl der Testpersonen erfolgte auf der Basis einer Quotenstichprobe aus einer Grundgesamtheit von 68.8 Millionen Personen (deutsche Bevölkerung ab 16 Jahren - Alkoholersterwerbsalter), die durch Interviewer persönlich und vor Ort befragt wurden. Die Felderhebung $\mathrm{zu}$ dieser Studie führte die Gesellschaft für Konsumforschung (GfK-Omnibussystem) in Nürnberg durch. Die Auswertung der gewonnenen Informationen erfolgte durch die Professur für Marktforschung der Hochschule Geisenheim University.

Die Repräsentativbefragung erlaubt es, die Ergebnisse auf die Grundgesamtheit zu übertragen. Nach den Erkenntnissen von Szolnoki und Hoffmann (2013) kann nur eine persönliche Vor-Ort-Befragung einer zufällig ausgewählten Stichprobe zu repräsentativen Ergebnissen für die gesamte erwachsene Bevölkerung führen. Internetbefragungen führen zu Verzerrungen der Ergebnisse zugunsten der regelmäßigen Weinkonsumenten. Die selten Weinkonsumierenden und wenig involvierten Verbraucher kommen in internetbasierten Stichproben wesentlich seltener vor.

\section{Ergebnisse}

\subsection{Konsumhäufigkeit}

Offizielle Statistiken zeigen, dass der Weinkonsum sich in den letzten Jahren in Deutschland nicht stark verändert hat. Der Deutscher Weinbauverband geht für 2017 von einer Gesamtweinmenge von ca. 20 Millionen hl aus (DWI, 2019). Auch der Anteil von Weintrinkern und Nicht-Weintrinkern ist in den letzten Jahren ziemlich stabil geblieben. Insgesamt $43 \%$ aller potentiellen Weintrinker ab 16 Jahren in Deutschland konsumieren keinen Wein. Überraschenderweise wird die Ablehnung von Weinkonsum in der Hälfte der Fälle mit dem Geschmack des Weines begründet, was Inhalt künftiger Untersuchungen sein wird. Auch die Anzahl der Häufig, Gelegentlich- und Selten-Trinker ist im Vergleich zu den Ergebnissen der 2014er und 2016er Befragungen mehr oder weniger unverändert geblieben (Szolnoki und Hoffmann, 2014a). Geht man davon aus, dass die Grundgesamtheit 68.832.000 Personen der Wohnbevölkerung in Privathaushalten umfasst - potentielle Weintrinker ab 16 Jahren -, kann man die genaue Größe der Häufigkeitssegmente errechnen: Häufig-Trinker 9.6 Mio., Gelegentlich-Trinker 15.1 Mio., Selten-Trinker 14.5 Mio. und Nicht-Weintrinker 29.6 Mio. Konsumenten (Abb. 1).

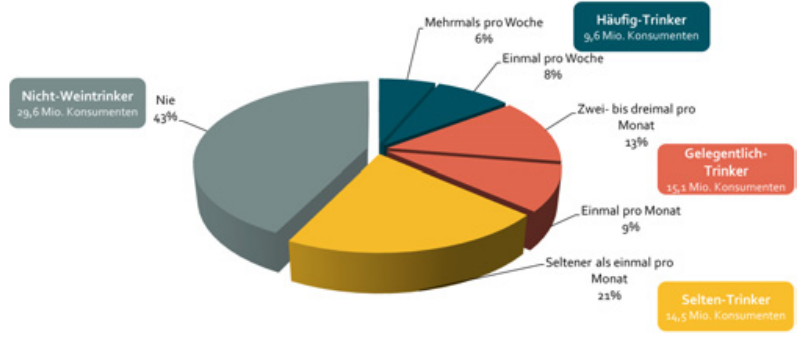

Abb 1. Konsumhäufigkeit von Wein $(n=2.063)$

*Häufig-Trinker $=$ trinken $\quad$ min. einmal/Woche Wein; Gelegentlich-Trinker $=$ trinken ein- bis dreimal/Monat Wein; Selten-Trinker $=$ trinken seltener als einmal/Monat Wein.

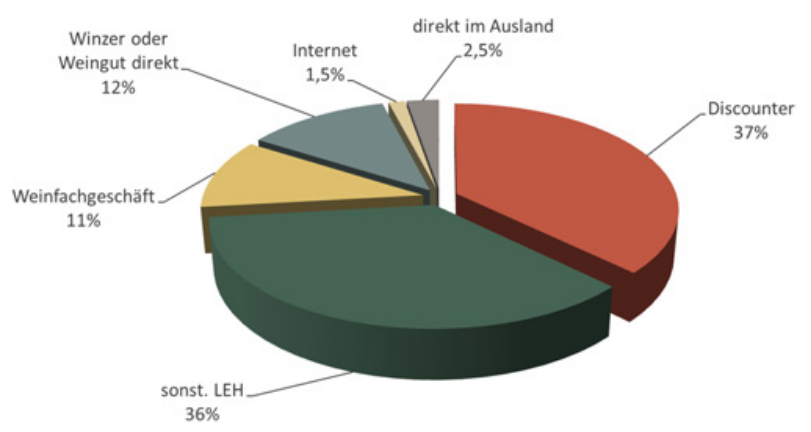

Abb 2. Nutzung der Einkaufsstätten für Wein $(n=1.181)$.

Bei Sekt stellt man ein komplett anderes Konsummuster fest: Der Anteil der Nicht-Sekttrinker liegt lediglich bei $7 \%$. Es konsumieren, zumindest gelegentlich, deutlich mehr Menschen in Deutschland Schaumwein (64 Mio.) als Stillwein (39 Mio.). Jedoch liegt der Anteil der SeltenTrinker (59\%) deutlich höher als bei Stillwein (21\%). Damit lässt sich Sekt als Anlass-Getränk definieren, das nur von einem kleinen Segment von $4 \%$ regelmäßig konsumiert wird. Im Gegensatz dazu weist Bier als alkoholisches Getränk einen deutlich höheren Anteil an Häufig-Trinkern auf. Fast jeder Zweite in Deutschland trinkt mindestens einmal pro Woche Bier. Dies entspricht einer Konsumentengruppe von 29.6 Mio. Personen über 16 Jahren. Die Ablehnung von Bier liegt bei $26 \%$.

\subsection{Nutzung von einkaufsstätten}

Einkaufstätten ohne Bedienungsservice machen mengenmäßig ca. 2/3 des Gesamtmarktes aus. Dies bedeutet, dass zwei von drei Flaschen im Handel über Discounter oder Supermärkte bzw. den Großhandel abgesetzt werden. Der Anteil von Fachgeschäften und Ab-Hof-Verkauf beläuft sich insgesamt auf 1/3 der vermarkteten Gesamtmenge (Abb. 2).

Die Ergebnisse der Studie zeigen, dass deutsche Konsumenten $37 \%$ ihrer Weine in Discountern und $36 \%$ in sonstigen LEHs kaufen. Blickt man auf die letzten Jahre zurück, ist eine Steigerung im Bereich sonstige LEH zu verzeichnen. Damit bewegen sich, unserer Berechnung nach, Supermärkte mengenmäßig auf dem gleichen Level wie Discounter. Der Anteil an Einkäufen in Weinfachgeschäften liegt bei $11 \%$, während Konsumenten ca. $12 \%$ ihrer Weine direkt bei Winzern und Weingütern kaufen. Im Vergleich zu den Befragungen in den Jahren 2014 und 2016 sind die Zahlen für diese beiden Einkaufsstätten etwas zurückgegangen (Szolnoki 


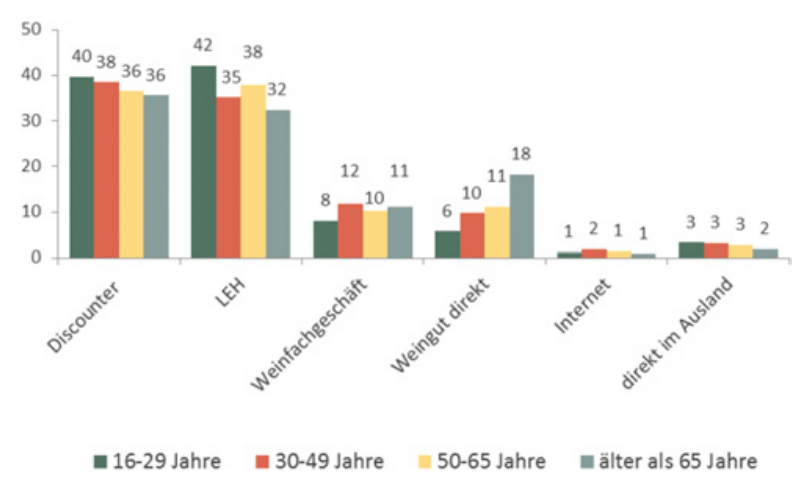

Abb 3. Nutzung der Einkaufsstätten nach Alter $(n=1.181)$.

und Hoffmann 2014a). Das Internet hat in den letzten vier Jahren an Bedeutung gewonnen.

\subsection{Segmentspezifische auswertung}

Die oben beschriebenen Ergebnisse zeigen lediglich die allgemeine mengenmäßige Verteilung auf die Einkaufsstätten, geben jedoch keinen detaillierten Einblick über die Bedeutung der Kundensegmente. Da zu vermuten ist, dass Untergruppen wie Männer oder Frauen und jüngere oder ältere Konsumenten ihre Weine auf unterschiedliche Art und Weise beziehen, wurde auch eine segmentspezifische Analyse durchgeführt, um herauszufinden, welche Untergruppen welche Einkaufsstätten für ihren Weinkauf präferieren. Vergleicht man das Geschlecht der Weinkäufer, wird deutlich, dass Frauen etwas häufiger Wein in Discountern kaufen als Männer. Dies liegt vermutlich daran, dass Frauen den täglichen oder wöchentlichen Lebensmitteleinkauf in Discountern häufiger mit Weineinkauf verbinden. Männer hingegen erreichen einen höheren Anteil in Fachgeschäften und $\mathrm{Ab}-\mathrm{Hof}$ und kaufen mehr Wein bei einer Auslandsreise.

Größere Unterschiede zeichnen sich bei der Nutzung der Einkaufsstätten nach Alter ab. Jüngere Konsumenten kaufen etwas mehr Wein in Einkaufsstätten mit niedrigerem Durchschnittspreis, was auf den etwas kleineren Geldbeutel in diesem Alter zurückzuführen ist. Der Anteil von Ab-Hof-Kauf steigt erst mit zunehmenden Alter. Demnach werden $18 \%$ aller Weine in der höchsten Alterskategorie direkt beim Weingut gekauft, während der Anteil bei den jüngsten Konsumenten lediglich bei $6 \%$ liegt. Jüngsten Weintrinker kaufen auch signifikant weniger Wein (8\%) in Fachgeschäften (Abb. 3).

Sucht man nach weiteren Faktoren, die die Nutzung von Einkaufsstätten signifikant beeinflussen, stößt man auf eine Variable von soziodemografischem und ökonomischem Charakter, und zwar die soziale Klasse, die anhand des höchsten Schulbildungsabschlusses, des derzeitigen Berufs und Netto-Einkommens definiert wird (Abb. 4). Verbraucher aus der Oberschicht kaufen weniger als ein Drittel ihres Weins in Discountern, während die Unterschicht fast jede zweite Weinflasche dort beschafft. Insgesamt kaufen Weintrinker aus der Unterschicht durchschnittlich $84 \%$ ihres Weines in Discountern und Supermärkten.

Der Anteil an gekauften Weinen in Weingeschäften und direkt vom Weingut ist dafür in der Oberschicht deutlich höher (18\% und 13\%). Auch im Bereich online

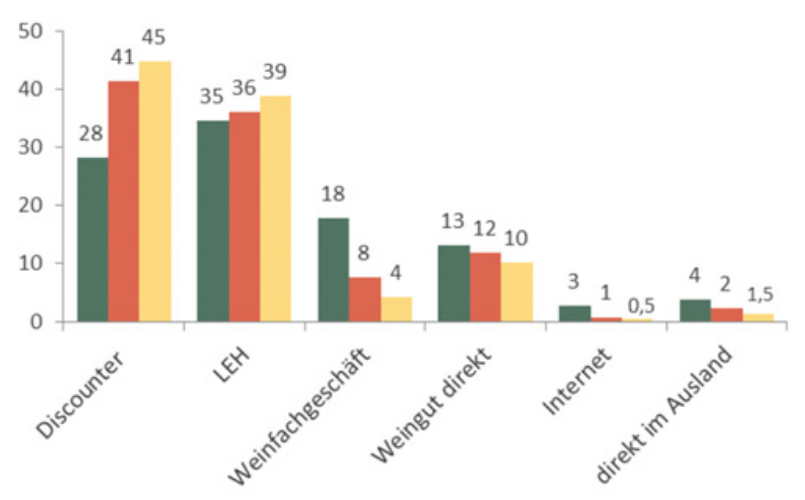

Oberschicht $\quad$ Mittelschicht $\quad$ Unterschicht

Abb 4. Nutzung der Einkaufsstätten nach sozialen Klassen $(n=1.181)$.

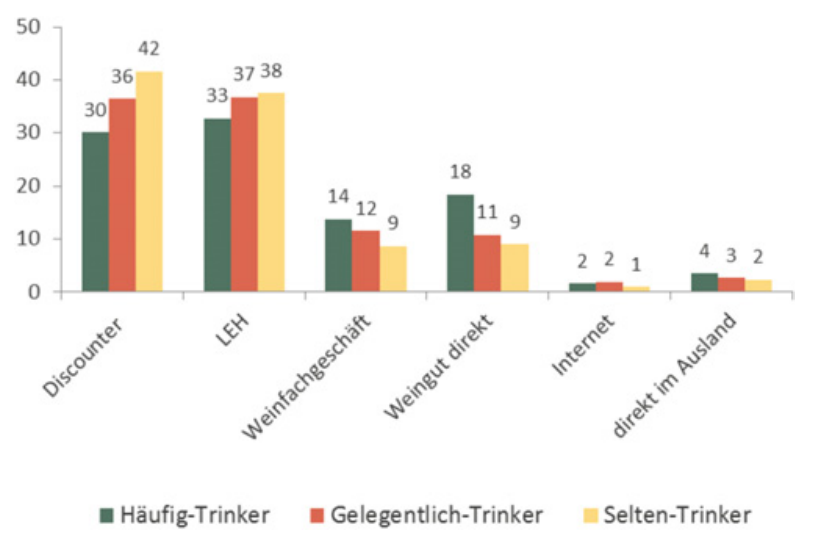

Abb 5. Nutzung der Einkaufsstätten nach Konsumhäufigkeit $(n=1.181)$.

Weinkauf haben die Konsumenten aus der Oberschicht einen höheren Anteil. Kunden aus der Mittelschicht nehmen wiederum bei der Nutzung der verschiedenen Einkaufsstätten jeweils eine mittlere Position (zwischen Unter- und Oberschicht) ein.

Da wir auch aus anderen Studien wissen, dass der Weinkonsum mit steigendem sozialen Status zunimmt, erhalten wir bei der Analyse der Einkaufsstättennutzung nach Konsumhäufigkeit ein ähnliches Muster. Hier unterscheiden sich Häufig-Trinker von SeltenTrinkern signifikant. Unter den Häufig-Trinkern finden wir einen überdurchschnittlichen Anteil an Konsumenten aus der Oberschicht. Häufig-Trinker haben den höchsten Anteil am Direktverkauf (18\%) und kaufen auch in Weingeschäften deutlich mehr (14\%). Mit sinkender Konsumhäufigkeit steigt der Anteil an Weinkäufen in Discountern und Supermärkten. Die Gelegentlich-Trinker liegen, wie zuvor die Mittelschicht, zwischen den Seltenund Häufig-Trinkern (Abb. 5).

\subsection{Modell zum gesamtweinmarkt in deutschland}

Obwohl das Marktbeobachtungssystem in Deutschland gut funktioniert, gibt es Statistiken und Werte, die man aufgrund der erhobenen Daten nicht direkt ermitteln kann oder die aus verschiedenen Gründen auf einer anderen Basis errechnet werden sollen. Die Nutzung von Einkaufstätten und, damit einhergehend, die Bedeutung der Verkaufskanäle sowie die wirtschaftliche 
Tableau 1. Bedeutung der Einkaufsstätten nach Personen-, Mengen- und Wertanteil.

\begin{tabular}{|c|c|c|c|c|c|c|c|}
\hline & $\begin{array}{l}\text { Marktdurch- } \\
\text { dringung }^{1} \\
(\%)\end{array}$ & $\begin{array}{c}\text { Durchschnitts- } \\
\text { preis }^{2} \\
(€ / 1)\end{array}$ & $\begin{array}{c}\text { Durchschnitts- } \\
\text { preis }^{2} \\
(€ / 0.751)\end{array}$ & $\begin{array}{l}\text { Mengen- } \\
\text { anteil }^{3} \\
(\%)\end{array}$ & $\begin{array}{l}\text { Absatz }^{4} \\
\text { (Mio. hl) }\end{array}$ & $\begin{array}{l}\text { Umsatz }^{5} \\
(\operatorname{Mrd} . €)\end{array}$ & $\begin{array}{c}\text { Wert- } \\
\text { anteil }^{6} \\
(\%)\end{array}$ \\
\hline Discounter & 71 & 3.00 & 2.25 & 37 & 5.32 & 1.60 & 23 \\
\hline LEH & 78 & 4.10 & 3.10 & 36 & 5.18 & 2.12 & 30 \\
\hline Weinfachgeschäft & 38 & 10.00 & 7.50 & 11 & 1.54 & 1.54 & 22 \\
\hline $\mathrm{Ab}-$ Hof & 31 & 8.60 & 6.50 & 12 & 1.68 & 1.44 & 21 \\
\hline Internet & 8 & 10.00 & 7.50 & 1 & 0.28 & 0.28 & 4 \\
\hline
\end{tabular}

1. Marktdurchdringung zeigt die Anzahl der Weintrinker in \%, die mindestens 1\% ihrer Gesamtmenge an Wein in der jeweiligen Einkaufsstätte gekauft haben.

2. Theoretische Durchschnittspreise basieren auf den ermittelten Preisen von GfK und IRI.

3. Direkte Antwort der Befragten auf die Frage „Wie viel Prozent der von Ihnen in den letzten 12 Monaten eingekauften Weinmenge haben Sie wo gekauft?"“.

4. Kalkuliert auf der Basis des Gesamtabsatzes von 14 Mio. hl Wein (nur Stillwein und nur off-Trade).

5. Kalkuliert auf der Basis der Absatzmenge und des Durchschnittspreises der jeweiligen Einkaufsstätte.

6. Berechnet auf der Basis der Wertschöpfungsdaten.

Bedeutung unterschiedlicher Untergruppen aufgeteilt nach Geschlecht, Alter, sozialer Klasse und Konsumhäufigkeit sind zwei typische Beispiele (Szolnoki und Hoffmann, 2014b).

Dieser Artikel bildet den Gesamtweinmarkt basierend auf Angaben der Endkunden ab. Mit diesem Ansatz wird der Markt von der Nachfrageseite her modelliert. Da Hochrechnungen immer theoretische Zahlen und Größen beinhalten, muss man erwähnen, dass es sich um Schätzungen handelt, die Abweichungen von der Realität aufweisen können. Jedoch muss hinzugefügt werden, dass man in Fällen, in denen die Realität gar nicht abbildbar ist, nur mit Hochrechnungen und Modellen arbeiten kann.

Aufgrund der Tatsache, dass in Deutschland verschiedene Marktforschungsunternehmen unterschiedliche Durchschnittspreise für Wein ermitteln, lassen sich die Menge und der Wert der Weine unterschiedlicher Einkaufsstätten für Stillwein in Deutschland nicht einfach quantifizieren. Mithilfe einer auf den unterschiedlichen Durchschnittspreisen basierenden Umrechnung werden bei zwei Einkaufsstätten (Discounter, sonstige LEHs) neue Mittelwerte gebildet. Diese neu kalkulierten Durchschnittspreise - vor allem für Discounter und andere Lebensmitteleinzelhändler - stellen im Grunde genommen eine Art Versuch des Mittelwegs dar. Die Durchschnittspreise für Weinfachgeschäfte, Ab-Hof-Verkauf und Internet wurden auf Basis von Experteninterviews und eigenen Recherchen definiert. Mithilfe der Durchschnittspreise und der von den Konsumenten angegebenen Anteile in den unterschiedlichen Kanälen, konnte der Gesamtabsatz und Gesamtumsatz für die jeweiligen Einkaufsstätten errechnet werden (Table 1).

Die Marktdurchdringung zeigt, dass Discounter und sonst. LEHs von 70-80\% aller Weintrinker für Weineinkauf genutzt werden. In diesen Einkaufsstätten liegt auch der Mengenanteil von Wein mit 36-37\% am höchsten. Die Befragten gaben an, 37\% ihrer Weine in Discountern und $36 \%$ in sonstigen LEHs zu kaufen. Diesen Verkaufskanälen wurden 2.25 bzw. $3.10 € / 0.751$ als Durchschnittspreis zugeordnet.

Der Anteil an Einkäufen in Weinfachgeschäften liegt bei $11 \%$, direkt bei Winzern und Weingütern bei $12 \%$. Im Vergleich zu den Befragungen in den Jahren 2014 und 2016 ist der Anteil dieser beiden Einkaufsstätten etwas zurückgegangen. In Fachgeschäften kaufen $38 \%$ der Kunden Wein, während der Ab-Hof-Anteil etwas niedriger bei $31 \%$ liegt. Beim Weinfachhandel und online
Geschäft wurde mit 7.50 und beim Ab-Hof-Verkauf mit $6.50 € / 0.751$ gerechnet.

Weinfachgeschäfte und Ab-Hof-Verkauf bewegen sich in allen Kategorien in einem ähnlichen Bereich, mit einer Ausnahme: Konsumenten, die bei Winzern und Weingütern direkt einkaufen, haben einen höheren ProKopf-Verbrauch und somit kommt dieser EinkaufsstättenTyp auf einen höheren Mengenanteil.

Als Basis für die Absatzmengen haben wir den geschätzten Hausverbrauch von 14 Mio. hl Stillwein in Deutschland zugrunde gelegt, der auf die unterschiedlichen Vermarktungskanäle verteilt wurde. Demnach setzen Discounter jährlich ca. 5.32 Mio. hl und sonst. LEHs 5.18 Mio. hl Stillwein in Deutschland ab. Im Fachhandel werden 1.54 und über Weingüter direkt 1.68 Mio.hl vermarktet. Der online Verkauf hat einen deutlich niedrigen Anteil von 0.30 Mio. hl, jedoch gehen wir davon aus, dass die Absatzmenge steigen wird, wenn die heutige, online-affine junge Generation älter wird und somit auch deren Weinkonsum zunimmt. Wertmäßig sind die Kategorien „Discounter, Weinfachgeschäft und Winzer/Weingut direkt" mit einem Anteil von 20-24\% ziemlich ausgeglichen. Die LEHs erreichten hier mit $30 \%$, was 2,12 Mrd. €Umsatz entspricht, den höchsten Anteil.

Aufgrund der hier ermittelten Mengen und Preise lässt sich ein Durchschnittspreis für den Gesamtweinmarkt in Deutschland von $4.99 € / 1$ und $3.75 € / 0.751$ errechnen. Basierend auf den oben genannten Mengen und Werten wird der Gesamtumsatz von Stillwein im deutschen Handel auf 7.0 Mrd. €geschätzt.

\section{Diskussion}

Die Breite und Tiefe des Angebotes und damit verbundene Dienstleistungs- und Serviceangebote haben sich in den letzten 15 Jahren stark verändert. Viele entdeckten das Potential und erweiterten ihr Weinsortiment, um mehr Kunden an sich zu binden. Discounter und sonstige LEHs machten sich stark im Bereich Wein und trotz der ohnehin hohen Vermarktungszahlen bei Wein, konnten sie ihre Position auf dem Weinmarktmarkt weiter ausbauen. Dies hatte negative Auswirkungen vor allen auf den Fachhandel, jedoch tauchen auch hier innovative Konzepte und neue Ansätze auf, um alte Kunden zurückzuholen oder neue Kunden zu gewinnen. Direktvermarktende Weingüter verzeichneten zwar in den letzten zehn Jahren einen 
leichten Rückgang, jedoch haben sie immer noch eine stabile Kundschaft.

Der Wettbewerb um die Kundenzustimmung geht allerdings weiter und wirft viele Fragen für die Zukunft auf: 1) Wie weit können Discounter und sonstige LEHs im Bereich Wein wachsen und welche Kunden nehmen die von ihnen angebotenen, weinbezogenen Dienstleistungen in Anspruch? 2) Können Fachgeschäfte Schritt halten und wie gut grenzen sie sich von den anderen Wettbewerbern ab? 3) Gib es eine Nachwuchsgeneration, die auch gerne Weine Ab-Hof kauft und inwieweit kann die Direktvermarktung von Weintourismus, der in den letzten Jahren an Bedeutung gewonnen hat, profitieren? Und 4) Wie verändert mittel- und langfristig die Digitalisierung des Weinverkaufs den Markt und was geschieht mit den Anbietern, die jetzt schon im online Geschäft aktiv sind?

Jeder Einkaufsstätten-Typ hat also seine Daseinsberechtigung und gleichzeitig auch seine Chancen und Risiken in der Zukunft. Die Konsumenten scheinen in diesem Spiel die Gewinner zu sein - sie bekommen für ihr Geld eine größere Auswahl, noch mehr Erlebnis und Dienstleistung. Es bleibt $\mathrm{zu}$ hoffen, dass die Vermarktungsstrukturen der Zukunft alle Akteure der Wertschöpfungskette zufriedenstellen und eine Win-Win Situation sowohl für Produzenten als auch Händler und Konsumenten entsteht.

\section{Literaturhinweise}

[1] Deutsches Weininstitut (DWI) (2019), Deutscher Wein Statistik 2018/19, www. deutscheweie.de

[2] G. Szolnoki, D. Hoffmann, Wine Econ. Policy 2, 57 (2013)

[3] G. Szolnoki, D. Hoffmann, Neue WeinkundenSegmentierung in Deutschland (GFFG Verlag, Geisenheim, 2014a)

[4] G. Szolnoki, D. Hoffmann, Int. J. Wine Business Res. 26, 27 (2014b)

[5] G. Szolnoki, Weinwirtschaft 26, 22 (2019) 